NASA Technical Memorandum $10288^{\overline{5}} 8$

\title{
Wind Tunnel Study of an Observatory Dome with a Circular Aperture
}

Gregory G. Zilliac and Ethan W. Cliffton

November 1990

(NASA-TM-10289B) WIND TUNNEL STUOY IF AN DASFRVATURY DOME WITH A CIRCULAR APERTURE (NASA) $19 P$ CSCL OBA

$N 91-15040$

Unclas

$63 / 890321120$ 


$$
\text { I... }
$$

5 : 


\section{Wind Tunnel Study of an Observatory Dome with a Circular Aperture}

Gregory G. Zilliac, Ames Research Center, Moffett Field, California Ethan W. Cliffton, MONOPTEC, San Francisco, California

November 1990

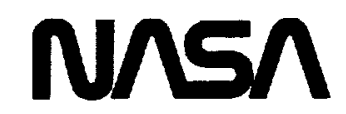

National Aeronautics and

Space Administration 
. 
"We see the opening of an era: it is an era

of seeking beyond the confines of our

atmosphere; may it be also an era of

awakening to the countries of our earth"

Bertrand de Jouvenel, circa 1970s

\section{SUMMARY}

This report describes results of a wind tunnel test of a new concept in observatory dome design, the Fixed Shutter Dome. From an aerodynamic standpoint, the new dome configuration is similar in overall shape to conventional observatory domes, with the exception of the telescope viewing aperture. The new design consists of a circular aperture of reduced area in contrast to conventional domes with rectangular or slotted openings. Wind tunnel results of a side-by-side comparison of the new dome with a conventional dome demonstrate that the mean and fluctuating velocity through the aperture and in the center of the new dome configuration are lower than those of conventional domes, thus reducing the likelihood of telescope flow-induced vibration.

\section{INTRODUCTION}

The best observatories are located well above the moisture laden lower atmosphere. At Mauna Kea, Hawaii, which stands in the Pacific air currents, the average wind conditions at the Keck Observatory are 10 to $30 \mathrm{mph}$ with gusts up to $125 \mathrm{mph}$ (see ref. 1). As new remote teleoperated observatories are developed at even higher altitudes, the subsequent exposure to high-altitude wind systems will be maximized. Winds in the upper part of the speed range, experienced by existing observatories (with conventional apertures), can significantly reduce viewing hours owing to flow-induced telescope vibration. In reference 2 , a new concept in dome aperture design is presented which uses a circular aperture. The circular aperture is intended to reduce the wind turbulence in the immediate vicinity of the telescope at all azimuths and elevations. This will allow for extended viewing programs, even in less favorable wind conditions, such as those due west at Mauna Kea, where objects rising in the east are tracked into the prevailing wind.

The Fixed Shutter Dome uses a split-sphere topology to allow the circular aperture to scan from horizon to zenith. Wind screens, which have been devised to protect telescopes in conventional slotted domes, are limited in strength and therefore must be abandoned at wind speeds well below those which the Fixed Shutter Dome can resist. 
The purpose of this test is to compare the aerodynamic behavior of a conventional slotted observatory dome configuration with that of a new configuration which uses a circular aperture of reduced area (see fig. 1). The primary goal of the comparison is to demonstrate that apertures of reduced area are likely to minimize the flow-induced vibration of telescopes housed within the dome.

A literature search turned up only one publication that is directly related to the present study. The effects of several shutter configurations on the aerodynamics of the Kitt Peak National Observatory Dome were studied in reference 3. Total aerodynamic torque on a dome model was used as an overall measure of the aerodynamic efficiency of the various shutter configurations. For each dome-shutter configuration, the torque was measured at several flow onset angles.

\section{Approach}

From an aerodynamic standpoint, the internal flow field in observatory domes can be fairly complex. The presence of a telescope and associated hardware provides sources for multiple flow interactions. For this reason, it was decided that the best way to quantify the benefits of the new dome design was to compare it to a dome that was geometrically similar in every aspect with the exception of the viewing aperture. It is likely that the presence of a telescope will alter the interior air flow from that reported here.

Four plexiglas wind tunnel models ( 8 in. in diameter) were used, three with circular apertures (angle of the aperture axis measured from vertical of 0,30 and $60^{\circ}$ ) and one with a conventional slotted opening as shown in figure 2 . These configurations represent a commonly used range-of-viewing in elevation. The diameter of the aperture was $3.125 \mathrm{in}$. The models were placed in the test section of a $32 \times 48$ inch wind tunnel in the Ames Research Center Fluid Mechanics Laboratory (fig. 3) and mounted on a micro-stepping motor-driven mechanism which was used to set the angle of the model relative to the freestream. The freestream velocity was $93 \mathrm{ft} / \mathrm{sec}$ and the Reynolds number based on dome diameter was $4.0 \times 10^{5}$.

A ring-wire anemometer (see ref. 4 and fig. 4) was used to measure the mean and fluctuating flow velocity at selected points in the interior of the spheres. Ring-wire probes are less sensitive to flow direction than conventional hot-wire probes, making them a valuable tool for velocity determination in flow fields where the flow angle relative to the probe is unknown and/or highly variable. The directional characteristics of the probe are shown in figure 5 . These results were obtained by setting the probe at a pitch angle, in the undisturbed freestream, and then rotating the probe through $120^{\circ}$ in $5^{\circ}$ increments. Like many probes, the response of the probe with flow angle is parabolic. The data show that the ring is not perfectly circular as indicated by the nonsymmetrical trend in the data at positive and negative sideslip angles. Calibration of the probe involved placing the undisturbed freestream flow of the $32 \mathrm{x}$ 
48 inch wind tunnel test section and then following standard King's law hot-wire anemometry calibration practices.

Precise positioning of the ring wire was difficult because of the limited accessibility of the interior of the dome and the curvature of the available probe mounting surfaces. The probe was supported by a structure made of 0.065 -in. steel rod which was attached to the lip of the dome aperture. Each new probe location required that the structure be deformed to achieve the desired probe orientation.

Measurements of the mean and fluctuating velocity were taken at points near the dome surface (in the aperture) and also at the center of the spherical part of the dome. Typically, the data near the aperture was obtained with the plane of the ring oriented to be coplanar with the local tangent of the dome surface and then translated inward slightly (0.5 in.) (likely position of a telescope secondary mirror).

The data system consisted of a Disa 55M01 hot-wire anemometer and a microVax II computer. Each datapoint is the result of an average of 2000 samples taken over a 4-sec sampling period.

Heating and ventilation systems of many full-scale observatory domes are designed to exchange the air at rates on the order of six times an hour. Air exchange rates of this magnitude are small in comparison to the flow rates through the aperture, hence they have been neglected in the present experiment. Aside from the viewing aperture, all other openings in the wind tunnel models were sealed.

\section{RESULTS}

As mentioned in the previous section, the ring-wire probe was placed either in the vicinity of the aperture, or at the center of the model, and the model was rotated in $5^{\circ}$ increments. Because the probe is attached to the model, it remains fixed relative to the model as the dome is rotated. Referring to figure 2 , the conventional dome position 1 is approximately the same location as that of the aperture measurements of the $30^{\circ}$ dome configuration. Likewise, position 2 is approximately the same location as that of the $60^{\circ}$ dome configuration aperture measurements. Again, the 30 and $60^{\circ}$ configuration aperture measurements were taken with the probe displaced radially inward $0.5 \mathrm{in}$. from the center of the opening. Measurements at position 3 were not taken during the present study.

One point to note when considering the data presented below is that since the only opening in the dome is the viewing port, the mass flow through this opening must be conserved. Hence through a portion of the aperture, the flow is entering the dome and through another portion, the flow is exiting.

Presented in figure 6 are the mean and fluctuating velocity variation with flow onset angle 
for the conventional dome at the two probe locations tested. The quantity $\theta$ defines the angular position of the model and $\theta=0$ when the aperture is precisely on the leeward side relative to the freestream direction. As expected, the data are nearly symmetric with respect to $\theta=180^{\circ}$ and the maximum mean flow velocity was measured with the rectangular opening facing directly into the freestream. The fluctuating velocity magnitude has maximums of nearly $10 \%$ of the freestream velocity and the absolute maximums occur when the aperture is on the leeward side of the body. With the aperture oriented into the freestream $\left(\theta=180^{\circ}\right)$ the fluctuations are also substantial.

If the probe is placed in the center of the spherical part of the dome with the axis of the probe aligned with the model axis of rotation, a comparison of figures 6 and 7 shows that, although the trends of the data are similar, the magnitude of the mean and fluctuating velocity are significantly reduced.

Shown in figure 8 are the results for the $30^{\circ}$ aperture model. Comparison with figure 6 ( $a$ and $b$ ) reveals that the circular aperture has the effect of reducing the peak mean velocity by approximately $30 \%$ and increasing the velocity fluctuations by $20 \%$ in the vicinity of the aperture. The $60^{\circ}$ dome configuration results (fig. 9) show trends that are different from the data presented to this point. Comparisons of figure 6 ( $c$ and $d$ ) with figure 9 show peak mean flow reductions of $60 \%$ and slightly reduced root mean square velocity fluctuations.

Measurements at the center of the spherical part of the $60^{\circ}$ dome (see fig. 10 and compare with fig. 7) showed greatly reduced mean and fluctuating velocity levels in comparison to similar measurements at the center of the conventional dome model.

\section{CONCLUSIONS}

Although it is difficult to conclude absolutely about the applicability of the results of scale model wind tunnel testing to full-scale, particularly when the full-scale structure will be significantly different (presence of telescope), the data presented herein support the following conclusions.

1. The mean and fluctuating velocities in the interior of a dome with spherical aperture are significantly less than that of a dome of conventional configuration under most conditions.

2. In the vicinity of the aperture, the mean and fluctuating velocity levels are approximately the same for a conventional dome aperture and a circular aperture. The velocity fluctuation level, near the circular aperture, drops off quite rapidly as the probe is moved towards the interior of the dome, while this is not the case with the conventional dome.

3. The data (and also flow visualizations not depicted here) show the optimum wind orientation to deliberately induce a flow of air into the dome through the aperture. This 
action would normally occur before a viewing session to bring the external and internal air temperatures into equilibrium thereby reducing local thermal convection.

4. Further study should be undertaken to measure the distribution of velocity along a radial line emanating from the center of the dome in the direction of the aperture. The design of the full-scale dome, with an actual telescope, would be enhanced by this knowledge, since there are distinct cost advantages to minimizing the radius of the dome.

The results of the side-by-side comparison of observatory dome apertures support the concept that a circular aperture of reduced area, in contrast to conventional domes with rectangular apertures, will, in all likelihood, reduce the level of telescope flow-induced vibration. 


\section{REFERENCES}

1. Kaufman, J; and Vecchioue, M.: A Summary of Nine Years of Weather Data from Mauna Kea Observatory. UCTMT Report No. 66, Aug. 1981.

2. Cliffton, E. W., U.S. Patent 4,840,458, 1989.

3. Hutchison, T.; and Skandalis, G.: Wind Tunnel Tests of 150-inch Telescope Dome Models with Various Shutter Configurations. University of Arizona Internal Report, 1966.

4. Giovanangeli, J. P., Chambaud, P.; and Mestayer, P.: Ring Wire Anemometer- Part I: Calibrations and Cooling Laws. Rev. Sci. Instrum., vol. 58, no. 11, 1987, p. 2172. 


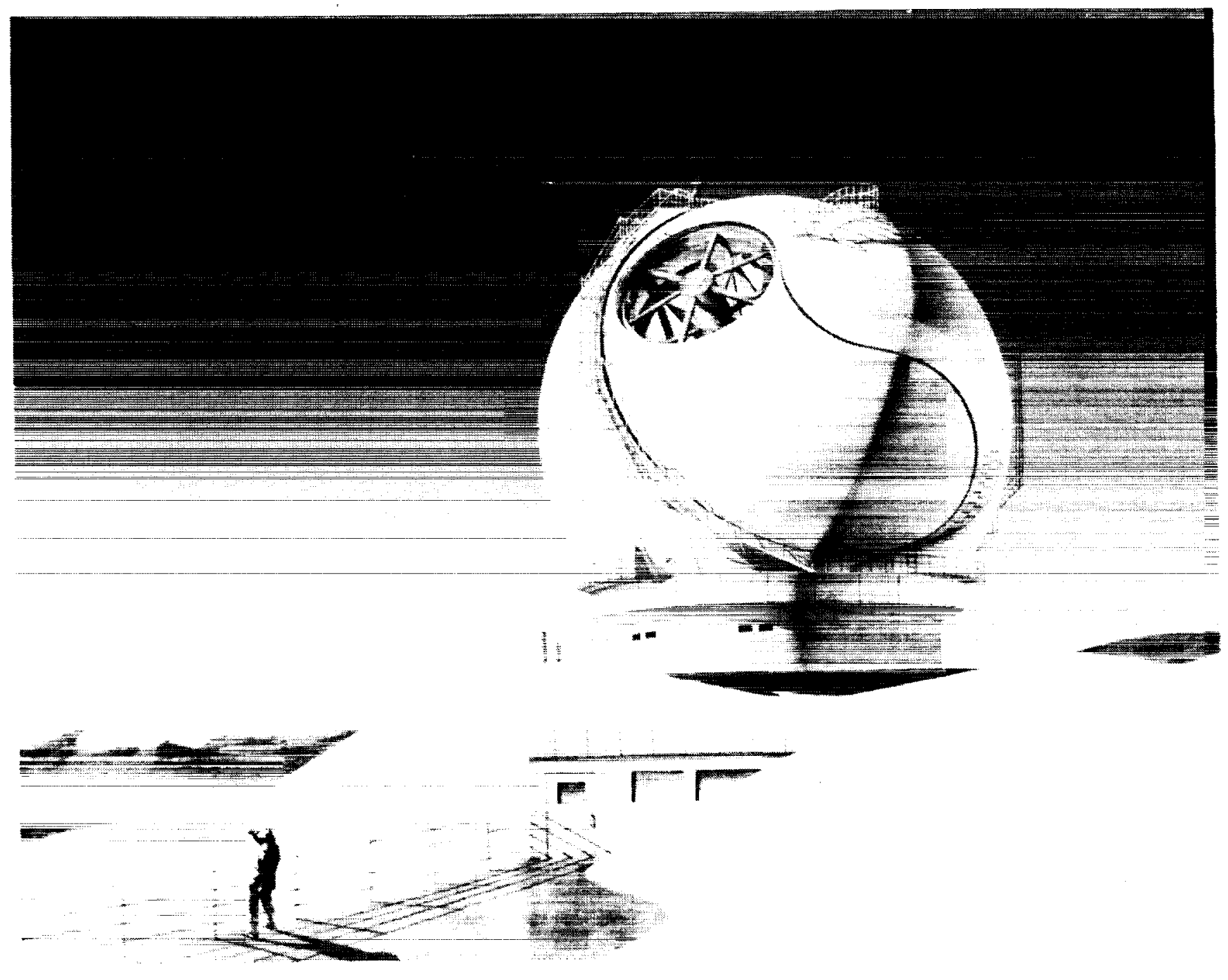

Figure 1. Artist's conception of the new dome configuration (ref. 2). 


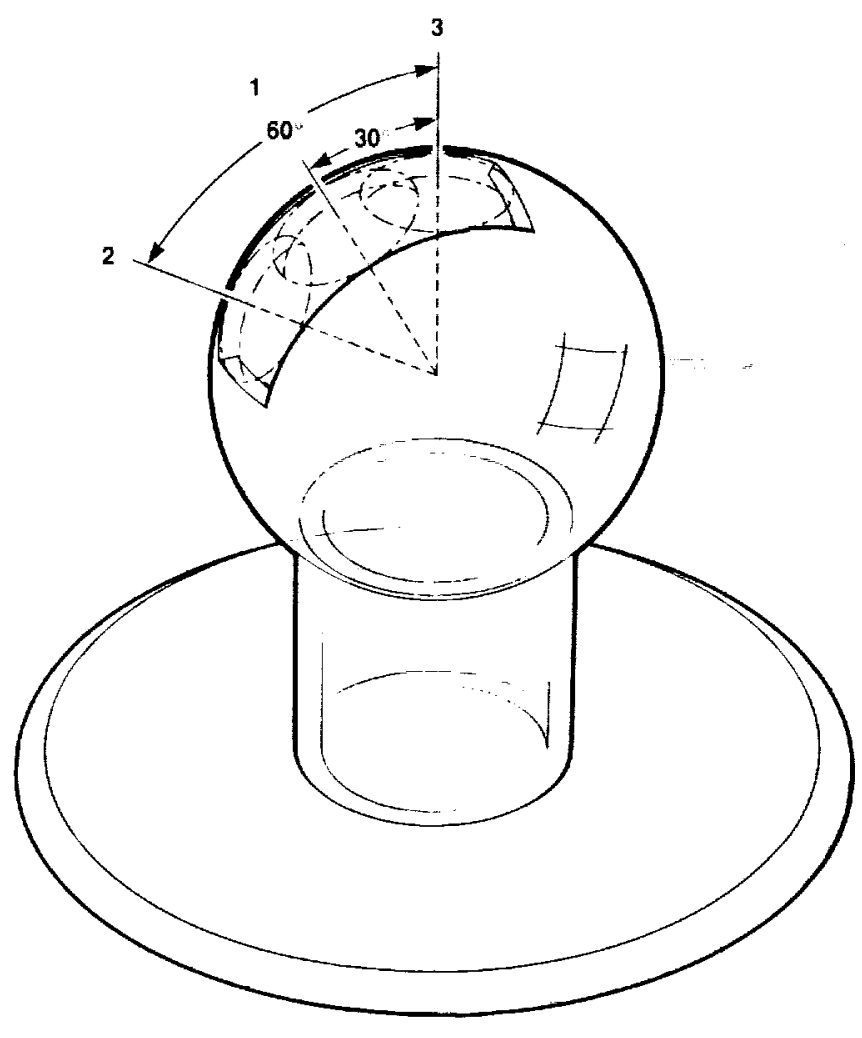

Figure 2. Superposition of the four wind tunnel model configurations tested. 


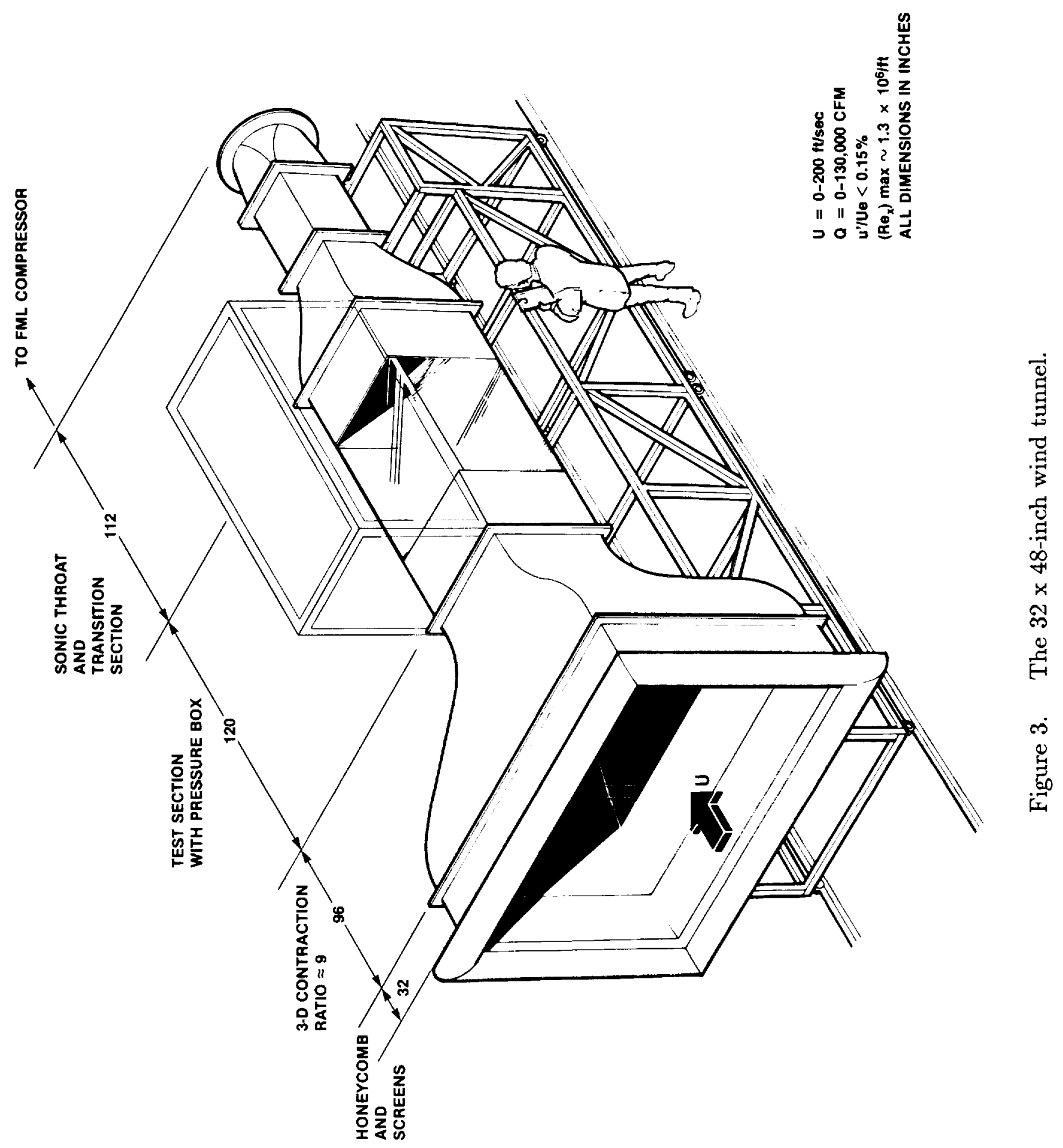




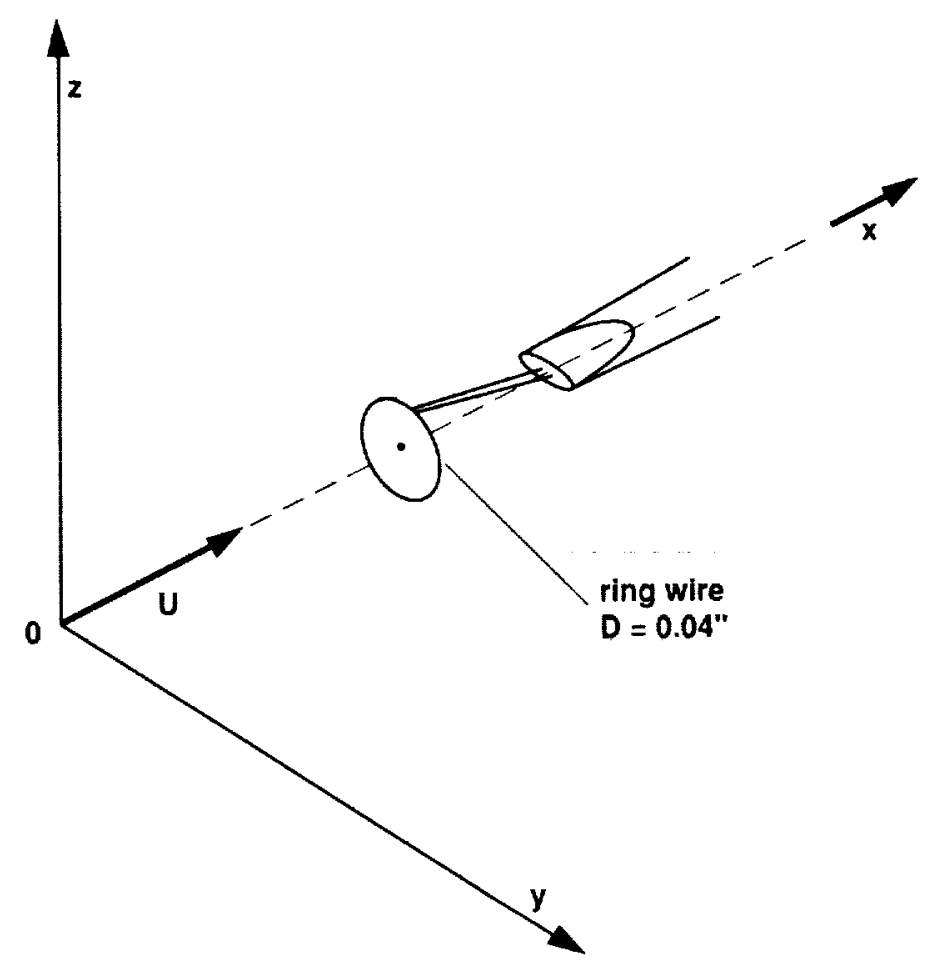

Figure 4. Ring-wire probe.

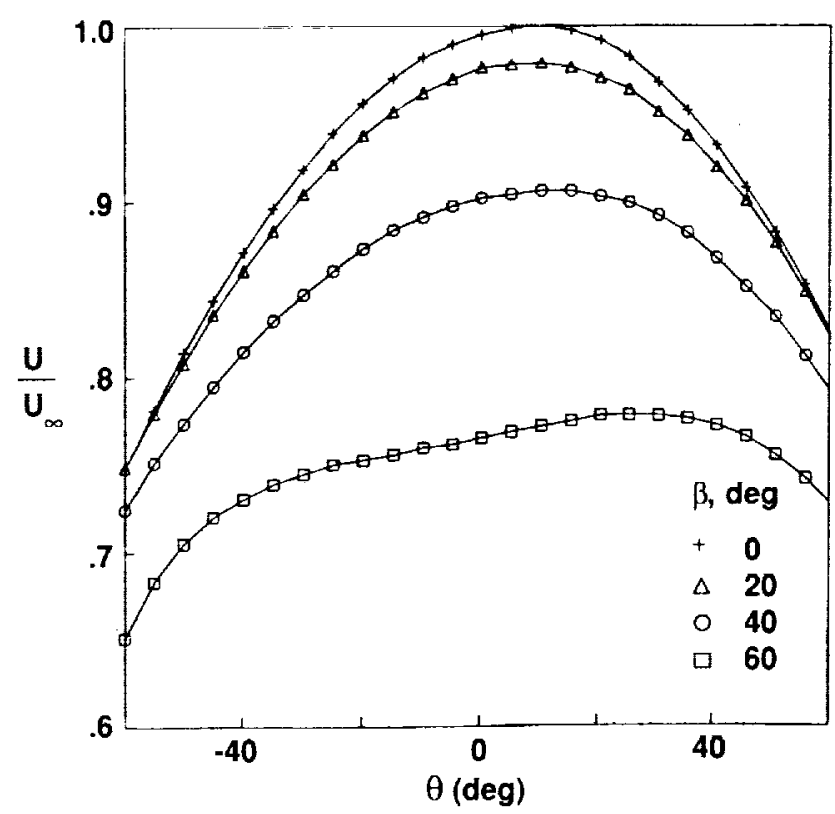

Figure 5. Ring-wire-probe directional characteristics. 

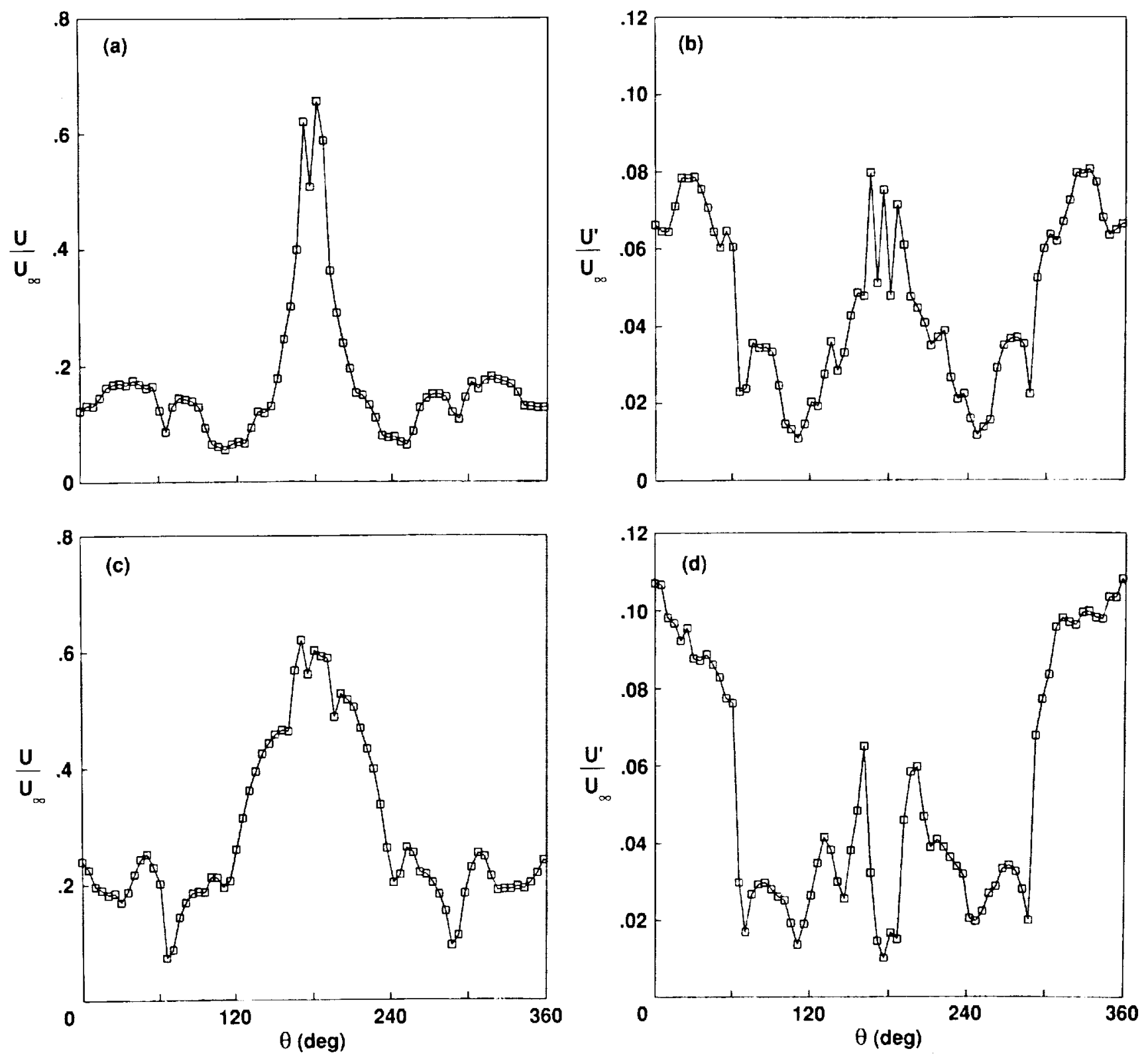

Figure 6. Conventional observatory dome flow measurements ner:- aperture.

(a) mean velocity at position 1 , (b) fluctuating velocity at position 1 ,

(c) mean velocity at position 2 , (d) fluctuating velocity at position 2 . 

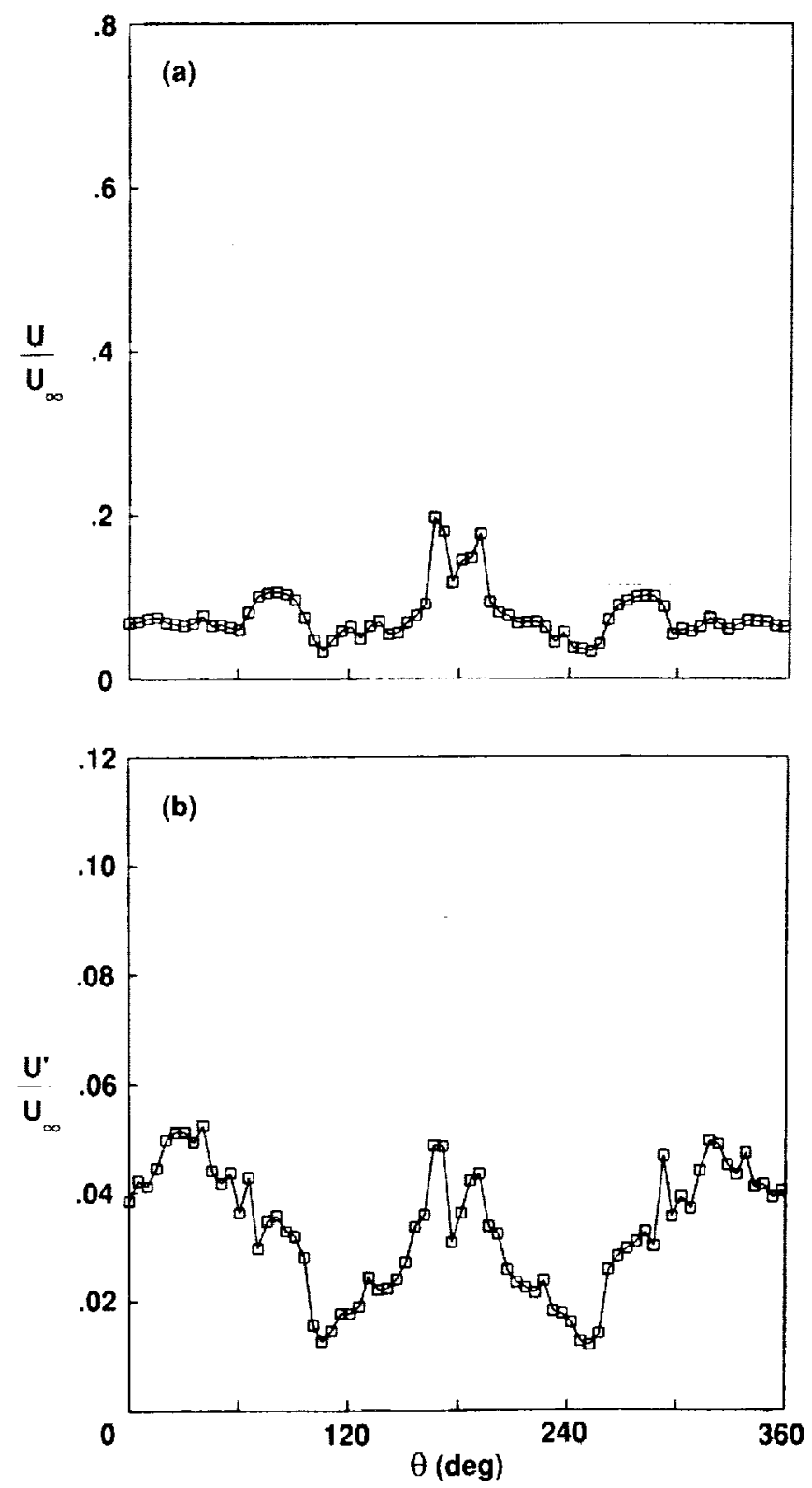

Figure 7. Conventional observatory dome flow measurements at dome center.

(a) mean velocity, (b) fluctuating velocity. 

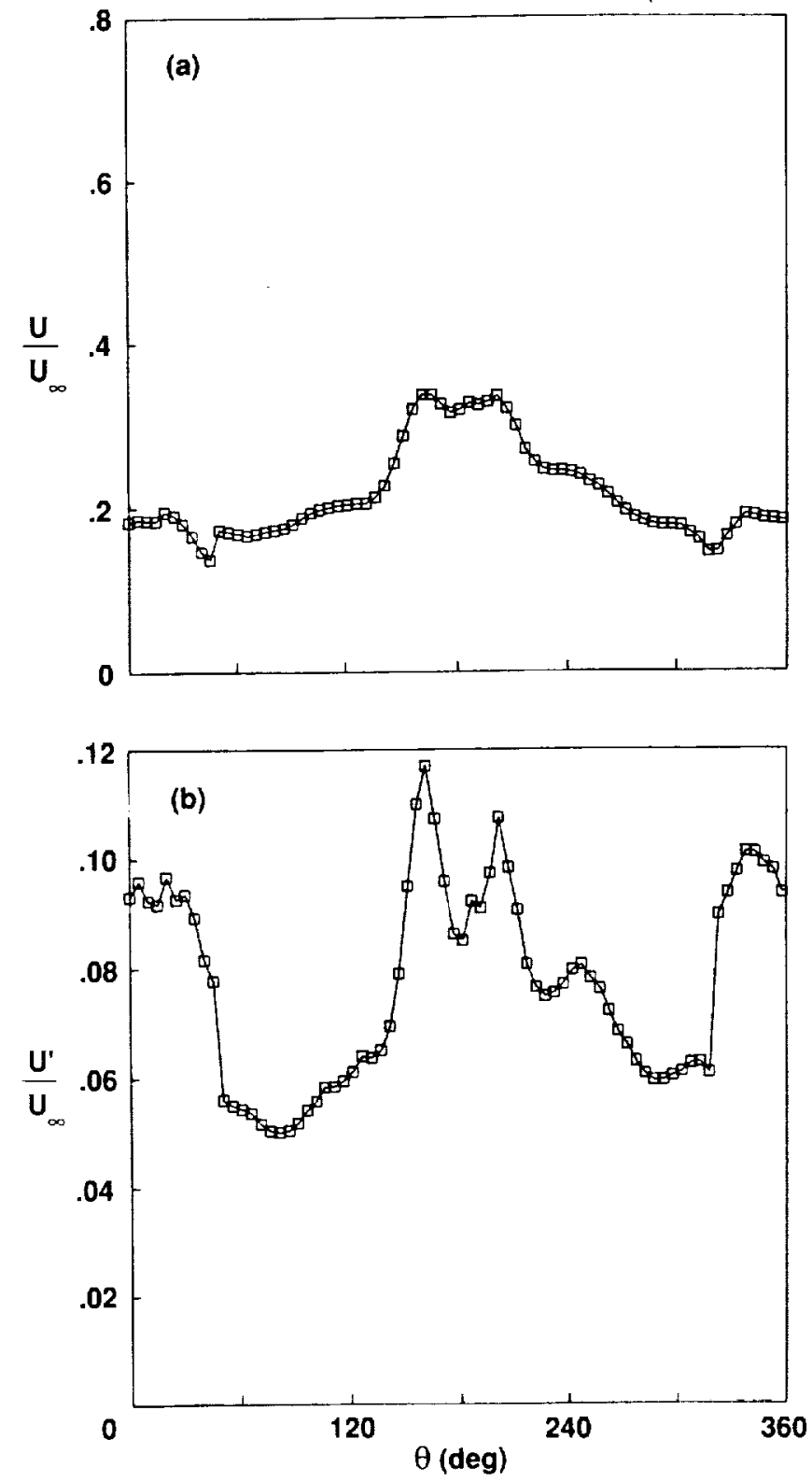

Figure 8. Observatory dome flow measurements near aperture $\left(30^{\circ}\right.$ model $)$. (a) mean velocity, (b) fluctuating velocity. 

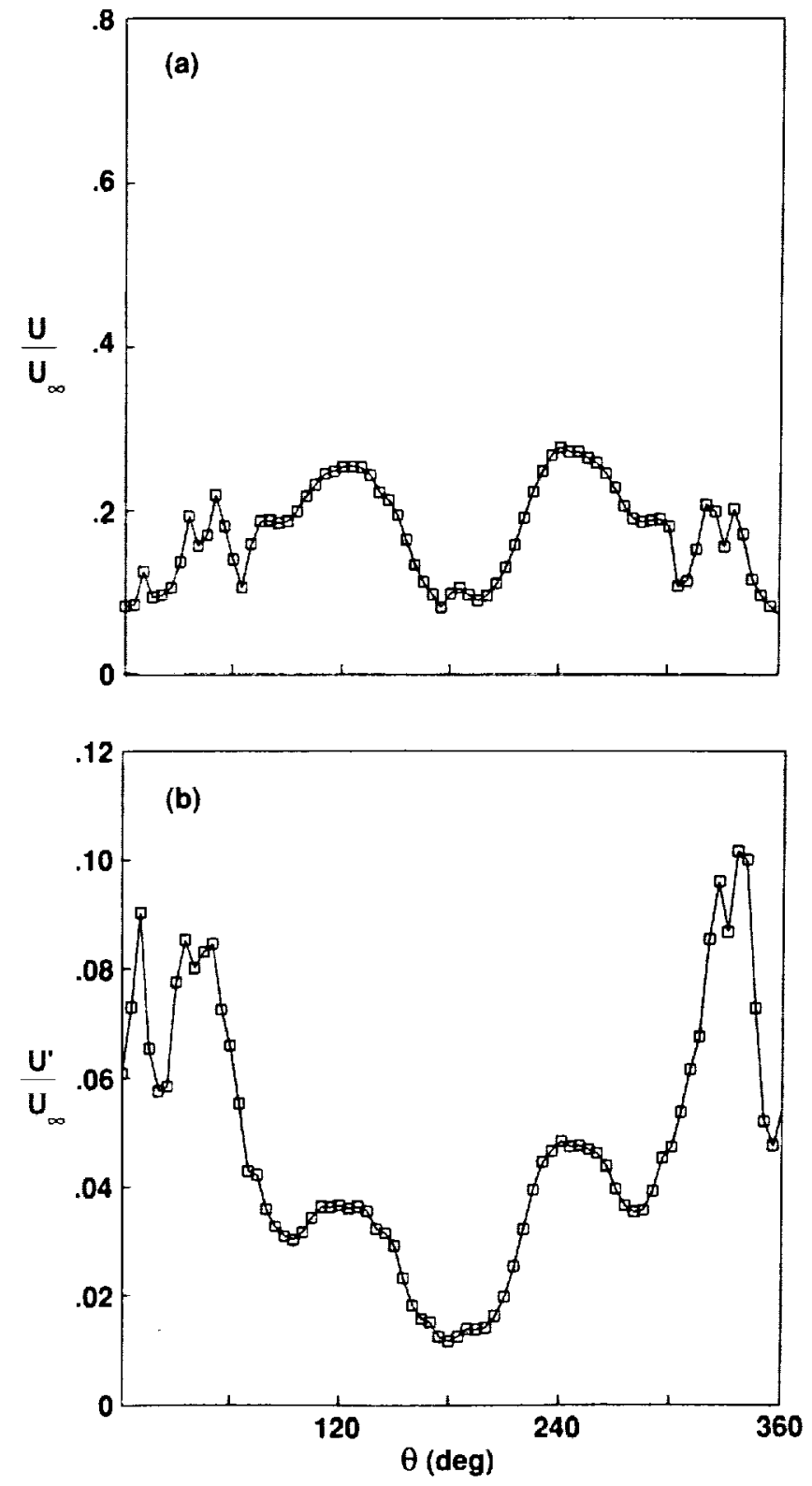

Figure 9. Observatory dome flow measurements near aperture $\left(60^{\circ}\right.$ model $)$.

(a) mean velocity, (b) fluctuating velocity. 

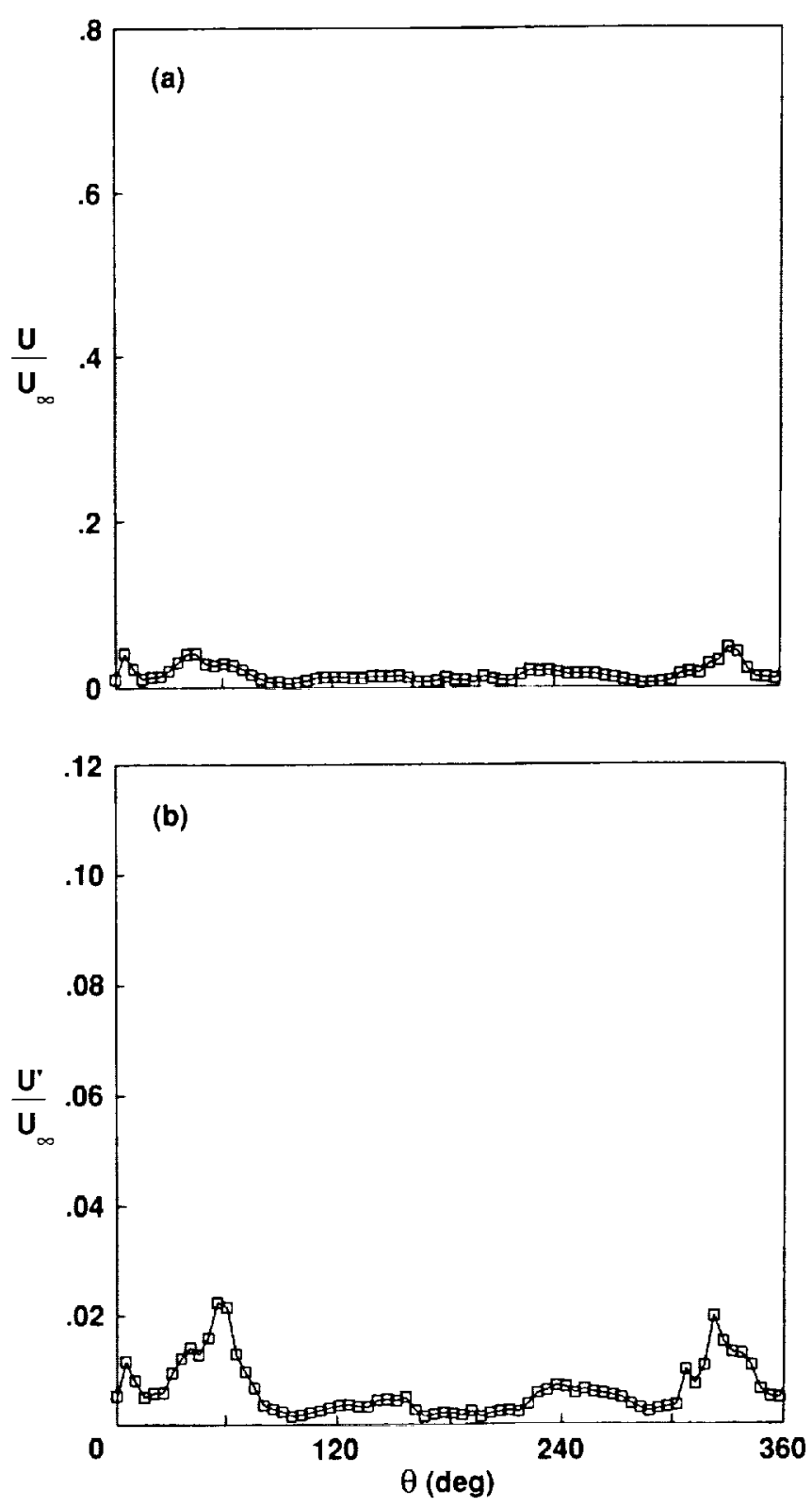

Figure 10. Observatory dome flow measurements at dome center $\left(60^{\circ}\right.$ model $)$. (a) mean velocity, (b) fluctuating velocity. 


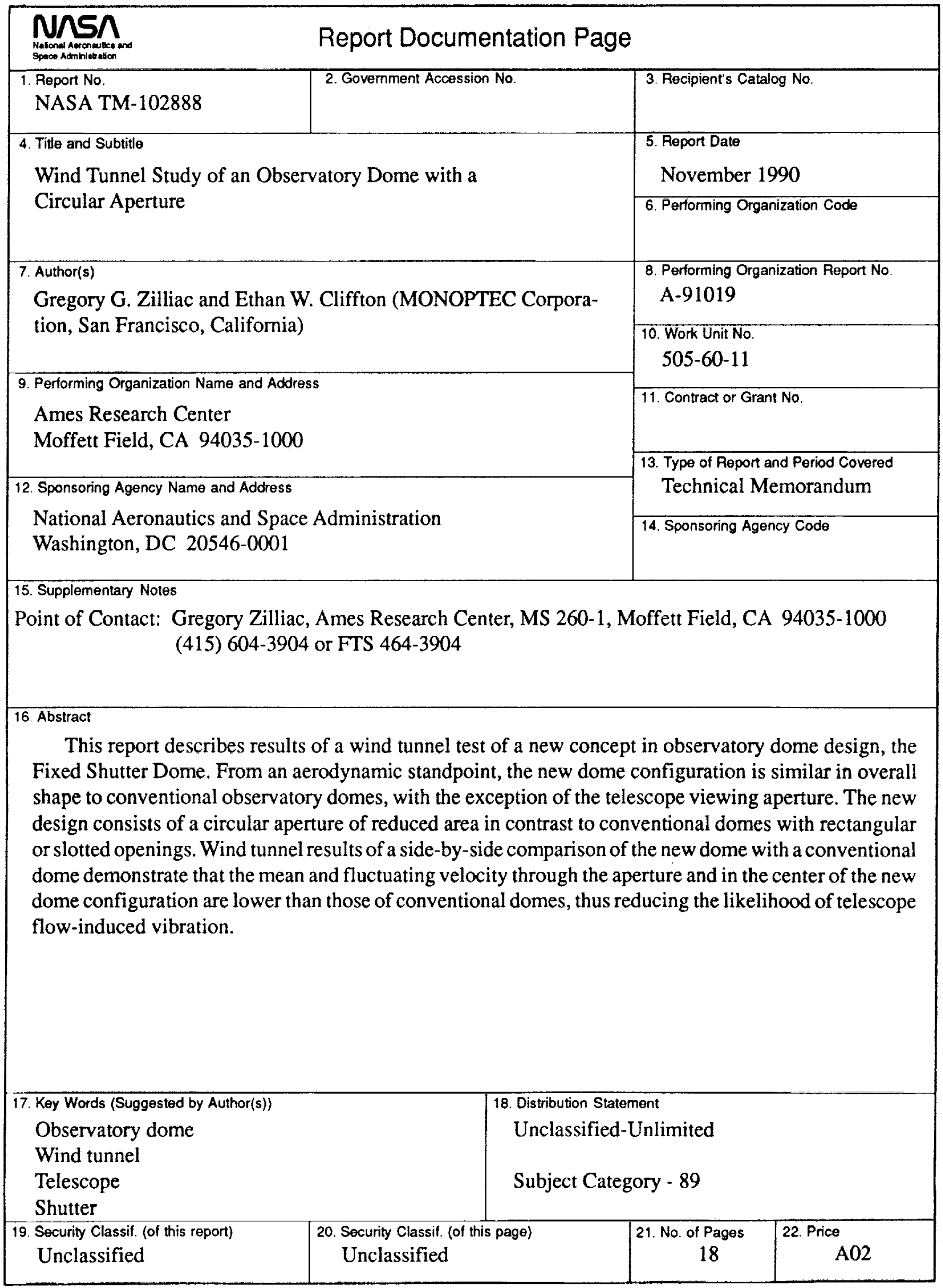

